\title{
Experimental study of the frictional heating phenomenon in elastomers
}

\author{
B. Pinedo ${ }^{1}$, M. Hadfield ${ }^{2}$, M. Conte ${ }^{3} \&$ A. Igartua ${ }^{1}$ \\ ${ }^{1}$ IK4 Tekniker, Tribology Unit, Spain \\ ${ }^{2}$ Bournemouth University, Faculty of Science and Technology, UK \\ ${ }^{3}$ Anton Paar, Switzerland
}

\begin{abstract}
The main limitation of polymers and subsequently of seals is their thermal resistance. Temperature rise on surfaces of bodies in contact due to frictional heating phenomena may influence considerably the tribological response and failure mechanisms of components. Moreover, high temperatures may cause structural changes of materials, as well as chemical reactions, oxidation and material degradation or melting. An experimental study of surface temperature rise of elastomers sliding against steel parts is herein presented. In this work, two of the most common polymers used in the sealing industry have been considered: NBR (Nitrile-Butadiene Rubber) and TPU (Thermoplastic Polyurethane). Surface temperatures and their evolution were measured during sliding of polymeric samples against steel cylinders by means of a high precision infrared thermo-camera. Friction coefficients were also acquired in order to study the effect of friction in relation to temperature rise. Moreover, steel cylinders with two different roughness and surface conditions were tested. Tests were carried out in absence of lubricant for an improved visualization of the temperature field. Test conditions and surface properties were selected so that they are inside the range of conditions recommended by seal manufacturers.

Keywords: frictional heating, polymer, contact temperature, roughness.
\end{abstract}

\section{Introduction}

Most of the frictional energy generated during sliding is converted into heat resulting in the temperature rise of surfaces. Considering that most of the polymers present a low temperature resistance, lower than $+300^{\circ} \mathrm{C}$, being able to 
estimate flash temperatures generated under actual working conditions of elastomeric components is a very important challenge. In previous studies by Conte et al. [1, 2], the authors dealt with the calculation of flash temperatures generated in polymers applying existing analytical models. In particular, the authors highlighted the importance of introducing friction coefficient evolution and non-constant thermal properties into the existing models in order to obtain more reliable results. In [3] Conte et al. studied analytically the frictional heating effects on polymers of different nature introducing experimental data obtained by means of tribological tests and thermal characterization techniques.

The main limitations to continue generating knowledge about the complex frictional heating phenomena occurring during sliding arise due to the existing difficulties to measure experimentally the temperatures at the contact. Many authors have previously used thermocouples, multi-function thin-film thermocouples and techniques involving the detection of IR radiation, among others [4]. Nevertheless, none of these techniques allows measuring the temperature rise at contact spots, and that it why up to now any technique has been widely accepted. The same happens with the theoretical models. Existing theoretical models are very useful to try to estimate the contact temperatures developed during sliding of bodies, however, they are based on several assumptions and none of them have been yet widely accepted. Furthermore, in the case of polymers there is a lacking of studied related to frictional heating effects.

In [5] Tzanakis et al. evaluated the flash temperatures developed in a fluoroelastomer composite tip seal sliding against a carbon steel plate under dry conditions. In this works the authors studied the effect of the sliding velocity, contact load and steel roughness on the contact temperature rise. They found that the sliding velocity plays a major role in local heating of surfaces and therefore, in the temperature rise of surfaces sliding against each other.

The aim of this work is to study the frictional heating phenomena on elastomers commonly used in the sealing industry sliding against steel parts under actual operating conditions. To this aim, NBR (Nitrile-Butadiene Rubber) and TPU (Thermoplastic Polyurethane) polymers have been tribologically tested by means of the TE77 (Plint) high frequency sliding test rig. Apart from friction coefficients, surface temperatures and their evolution were also measured during sliding of polymeric samples against steel cylinders by means of a high precision infrared thermo-camera. In order to analyze the effect of the steel parts roughness sliding against elastomeric seals, steel samples of different roughness have been tested.

\section{Experimental setup and specimens}

Several friction tests were carried out in the TE77 test rig represented in Figure 1. In order to reproduce the contact between an elastomeric seal and its metallic counter-part the cylinder on flat configuration shown in Figure 2 was chosen. The cylinder samples were made from a 42CrMo4V (F1252) steel, with a diameter of $10 \mathrm{~mm}$, a length of $10 \mathrm{~mm}$ and a hardness of $60 \mathrm{HRC}$. Flat samples 
have a length of $15 \mathrm{~mm}$ and they were manufactured from two type of elastomers commonly used within the sealing industry: NBR (Nitrile-Butadiene Rubber) and TPU (Thermoplastic Polyurethane).

Thermoplastic polyurethane elastomers (TPU) combine the mechanical properties of vulcanised rubber with the processability of polymers. It is formed by a combination of hard and soft segments. It can be repeatedly melted and processed due to the absence of chemical networks.

NBR (Nitrile rubber, Polybutadiene-acrylonitrile) is a thermoset elastomer that is both elastic and viscous. Typical rubber compounds consist of several ingredients added to improve physical properties, affect vulcanization, improve processability, and prevent long-term deterioration. Rubbers are vulcanised in order to form chemical bonds between elastomer chains, so that their stability, strength and resilience is increased.

The thermal resistance of both polymers under test is approximately $+110^{\circ} \mathrm{C}$.

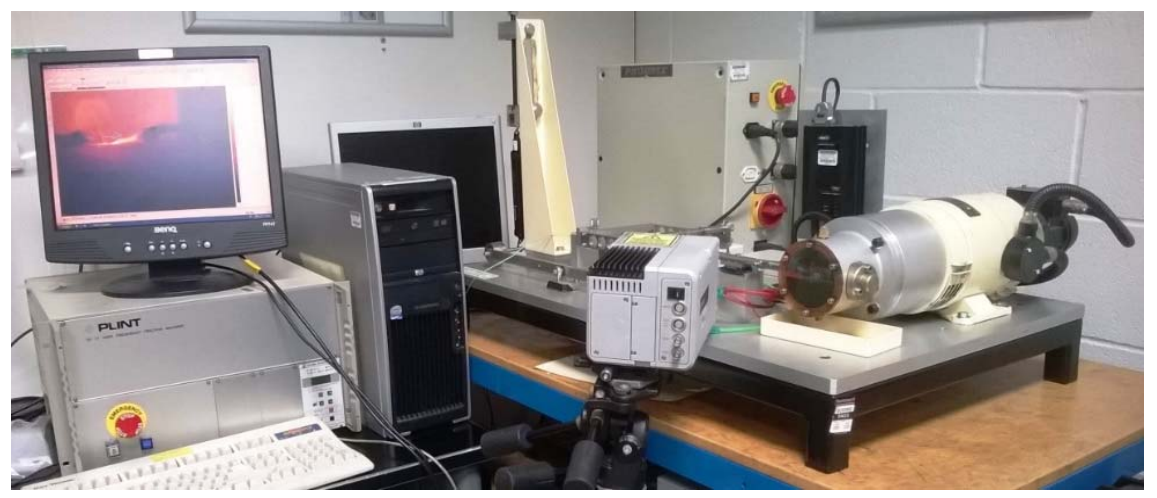

Figure 1: TE77 high frequency sliding test rig.

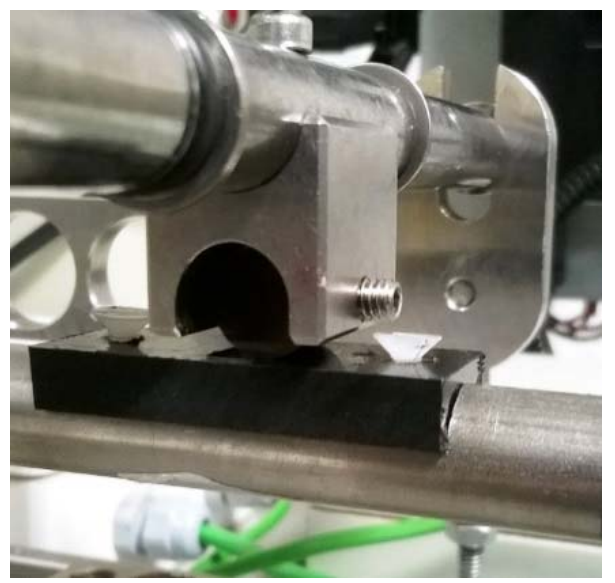

Figure 2: Cylinder on flat configuration. 
The steel cylinder is fixed in a holder connected to the system. The elastomeric sample moves in reciprocating motion actuated by means of a motor. The upper part is connected to a load cell which measured generated friction forces during the tests. As can be observed in Figure 1, a Flir SC300 high precision thermo-camera was installed close to the tribo-system in order to acquire surface temperature evolution during the tests. An infrared camera measures and images the emitted infrared radiation from an object. The intensity of the emitted energy from a specific object varies with temperature and radiation wavelength. The camera was installed at a distance of $100 \mathrm{~mm}$ from the polymer/cylinder contact in order to obtain an adequate focus. The thermal camera has a working range of $-20^{\circ} \mathrm{C}$ to $+2000^{\circ} \mathrm{C}$ with an accuracy of $\pm 1 \%$ or $\pm 1^{\circ} \mathrm{C}$ for measurement ranges up to $+150^{\circ} \mathrm{C}$. The system has a sensitivity of $20 \mathrm{mK}$ at $30^{\circ} \mathrm{C}$ and a resolution of $320 \times 240$ pixels. Calibration of the camera was carried out following the procedure described by manufacturers. In order to avoid possible errors arising from reflections, all the metallic parts of the system were properly insulated.

Friction tests were carried out at room temperature and under dry sliding conditions. A stroke of $5 \mathrm{~mm}$ was set, a normal load of $15 \mathrm{~N}$ and two different sliding velocities, $0.1 \mathrm{~m} / \mathrm{s}$ and $0.25 \mathrm{~m} / \mathrm{s}$. Coefficient of friction and surface temperatures were recorded for 10 minutes and each test was repeated a minimum of 2 times.

In order to study the effect of surface conditions on the generated friction and surface temperatures, steel cylinder with different Ra values were tested: $0.1 \mu \mathrm{m}$ and $0.5 \mu \mathrm{m}$.

\section{Experimental results}

Figure 3 shows a typical thermal image of the tribo-system taken during the tribological tests.

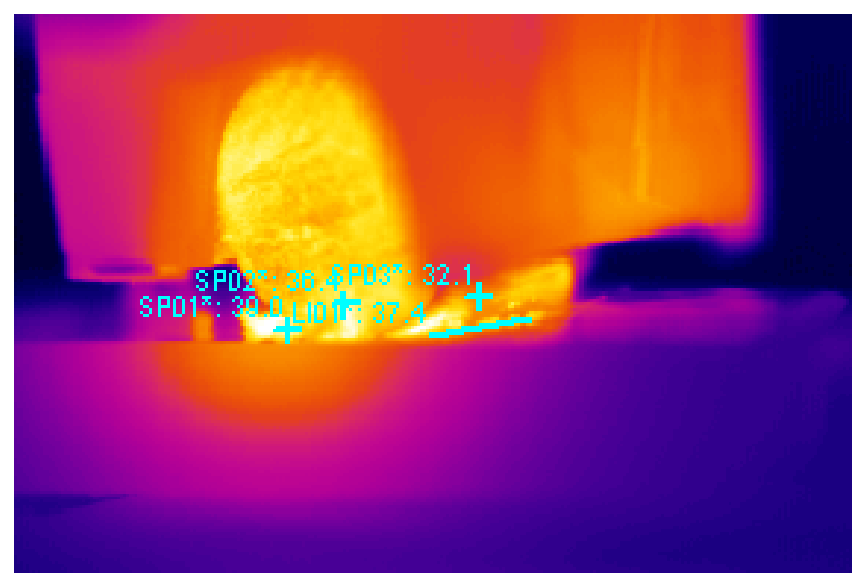

Figure 3: Thermal image example from the tribo-system. 
Figure 4 shows friction results obtained from the tribological tests of TPU samples sliding at different velocities against steel cylinders with different roughness.
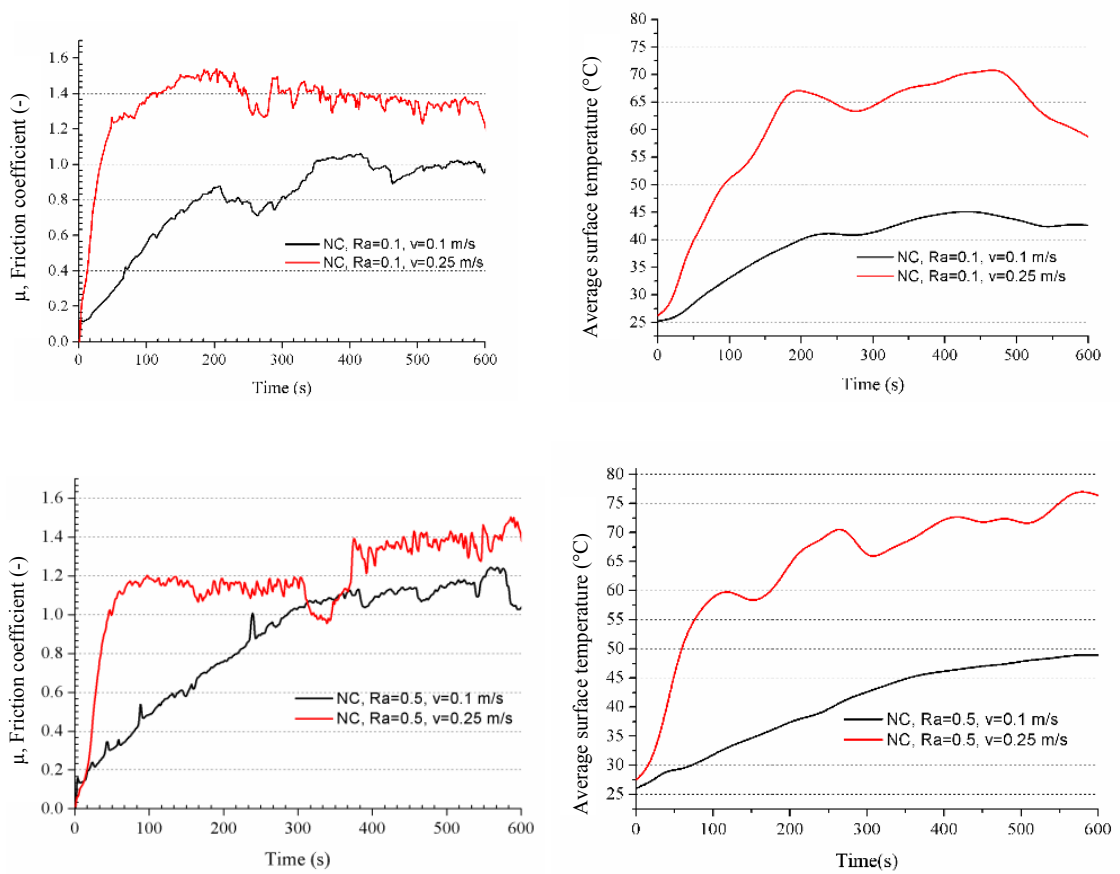

Figure 4: Friction force and temperature curves of the TPU at different sliding velocities and steel roughness.

As can be observed, both the friction coefficients and the surface temperatures of the elastomeric sample increase with the sliding velocity. In particular, for a cylinder with a Ra of 0.1 , the steady friction coefficient rises from 1 to 1.4 with a velocity increment of $0.15 \mathrm{~m} / \mathrm{s}$. The effect of increasing the sliding velocity on the temperatures reached at the surface of the TPU during the test is even greater than the effect of velocity on friction. Specifically in the case of the cylinder sample with a roughness of $\mathrm{Ra}=0.1$, the temperature rises from $+45^{\circ} \mathrm{C}$ to $+70^{\circ} \mathrm{C}$ when the velocity rises from $0.1 \mathrm{~m} / \mathrm{s}$ to $0.25 \mathrm{~m} / \mathrm{s}$.

The effect of the cylinder roughness on the developed friction is almost negligible at $0.25 \mathrm{~m} / \mathrm{s}$. At $0.1 \mathrm{~m} / \mathrm{s}$, however, the friction coefficient rises from 1 to 1.2 when the roughness rises from 0.1 to $0.5 \mu \mathrm{m}$. Therefore, it was found that increasing the roughness also affects the temperatures reached at the surfaces at both sliding velocities. In particular, at $0.1 \mathrm{~m} / \mathrm{s}$ the maximum surface temperature in the TPU surface rises from $+42^{\circ} \mathrm{C}$ to $+50^{\circ} \mathrm{C}$ when increasing the cylinder roughness from 0.1 to $0.5 \mu \mathrm{m}$. At $0.5 \mathrm{~m} / \mathrm{s}$ the effect of roughness is the same; a temperature rise of approximately $5^{\circ} \mathrm{C}$ was observed. Hence, it can be concluded 
that the friction and temperature rise increase monotically with steel parts roughness under the selected test conditions. These results are in good agreement with Guha and Chowdhuri [6]. It can be observed that, in general, the lower the sliding velocity the longer it takes to stabilize both friction and temperature curves. It is important to highlight that friction and temperatures curve tendency are in good agreement.

Figure 5 presents the variation of friction coefficients and surface temperature of the NBR for different velocities and cylinder roughness. It can be seen that in the case of the NBR generated friction also increases with velocity. At $0.1 \mathrm{~m} / \mathrm{s}$ the stable friction coefficient is between 0.8 and 0.9 in all the cases whereas at $0.5 \mathrm{~m} / \mathrm{s}$ it is between 1.1 and 1.2 . The effect of the velocity on the generated temperatures is even greater also in this case than the effect of velocity on friction. The average temperature measured at the surface of the NBR rises from $+40^{\circ} \mathrm{C}$ up to $+60^{\circ} \mathrm{C}$ under stable conditions when increasing the velocity.
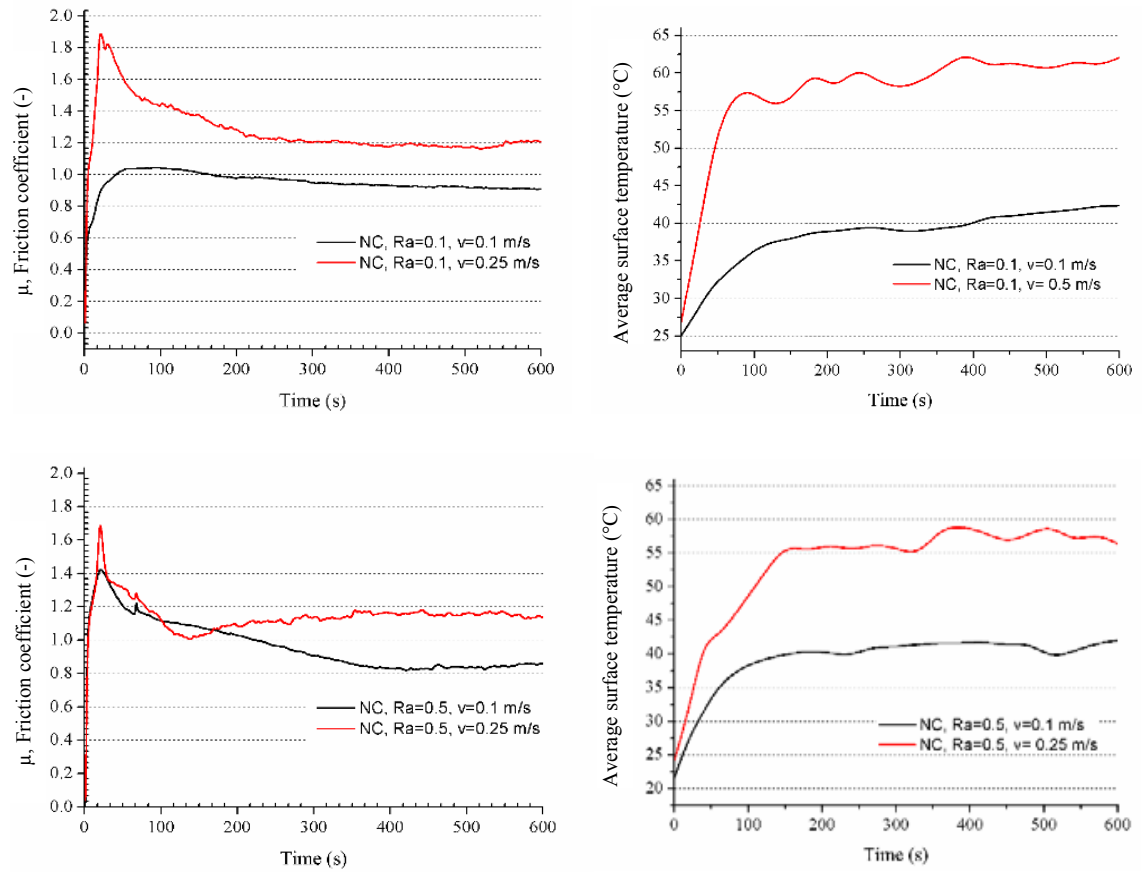

Figure 5: Friction force and temperature curves of the NBR at different sliding velocities and steel roughness.

Moreover, it was seen that the roughness of the steel cylinders does not affect the friction coefficient value under stable sliding conditions. Nevertheless, it was found that the cylinder roughness affects the evolution of friction during the running-in period and the form of the friction curve during the first sliding cycles (200s). The main reason for being almost independent the roughness and friction after some sliding cycles may be the severe operating conditions. In other words, 
even if the polymers under test work usually under lubricated conditions, in this case, for a better visualization of the temperatures at the surface of elastomers, tests were carried out under dry conditions. Thus, the absence of lubrication involves that the wear of samples is more so that after the first sliding cycles the effect of roughness ends up being negligible in all the cases.

Regarding the temperatures at the surface of the polymer, the effect of roughness on the steady temperatures was found also to be negligible comparing to the effect of velocity. In particular, at $0.1 \mathrm{~m} / \mathrm{s}$ surface temperatures of about $+40^{\circ} \mathrm{C}$ were measured at the polymer surface for both tested roughness values, and the temperature value rises up to $+60^{\circ} \mathrm{C}$ at a sliding velocity of $0.5 \mathrm{~m} / \mathrm{s}$.

TPU and NBR are very used to manufacture low velocity seals for hydraulic applications, and they both present similar upper thermal limits of about $+100^{\circ} \mathrm{C}$. Nevertheless, in this work it was found that both materials present very different performance under the same operating conditions. The behavior difference is evident at a first glance observing friction coefficient curves. As can be seen, the tendency of the friction curves are completely different. In the case of the NBR, the friction coefficient increases during the first running cycles up to reaching its maximum value. Afterwards, it decreases with time until reaching a steady state value. The maximum point was found to occur a bit before a steady sliding velocity is reached, and from this point on friction continuously decreases until a steady temperature is reached. In the case of the TPU, it does not present a maximum friction peak. The friction coefficients increases with time until a steady friction value is reached.

It was also found that during the tests with the TPU more noise and vibrations were generated. These observations are in good agreement with the acquired friction curves which present instability peaks during all the tests (Figure 4). The origin of such instabilities may be caused by the hardness of the material i.e. the TPU presents a hardness of 93 Shore A while the NBR presents a lower hardness of 70 Shore A. The hardness of materials was measured by a Shore durometer used to measure the hardness of polymers. In all the cases it was seen that the friction coefficient and the surface temperature increase rapidly during the first cycles. The frictional energy can be dissipated in several ways such as being transformed into vibrations, noise, material deformation or new material creation, or can be stored in the tribo-system. Hence, in order to analyse correctly a system it is necessary to study both friction and wear rates because two different materials may present the same friction but different mass loss under the same test conditions. Thus, it is necessary to calculate the specific wear rate to study how the energy is partitioned within and between the materials [7]:

$$
E_{w}=\frac{E}{\Delta m}=\frac{v N \int_{t_{i}}^{t_{f}} \mu(t) d t}{\Delta m}
$$

where $E_{w}$ is the specific wear rate, $E$ is the energy dissipated by friction, $v$ is the mean relative sliding velocity, $\mathrm{N}$ is the normal load, $\mu$ is the coefficient of friction, $t_{\mathrm{i}}$ the initial time, $t_{f}$ the time at the end of the test, and $\Delta m$ is the total mass loss.

Figure 6 presents the mass loss of the polymeric samples at different velocities when they are sliding against a steel cylinder with a Ra roughness of 
0.5. As can be seen, results showed that the mass loss or wear of the NBR is about 4 times bigger than the wear of the TPU under both $0.1 \mathrm{~m} / \mathrm{s}$ and $0.25 \mathrm{~m} / \mathrm{s}$. Hence, not only the mass loss of the Nitrile Rubber samples is bigger but also the contact width or wear scars measured after the tribological tests. Hence, it can be concluded that even if both polymers lead to similar friction coefficients or similar frictional work under the selected test conditions, the TPU shows a higher wear resistance i.e. the NBR presents a higher wear rate.

Moreover, it was found that roughness of the steel cylinder does not influence the mass loss of the polymeric samples.

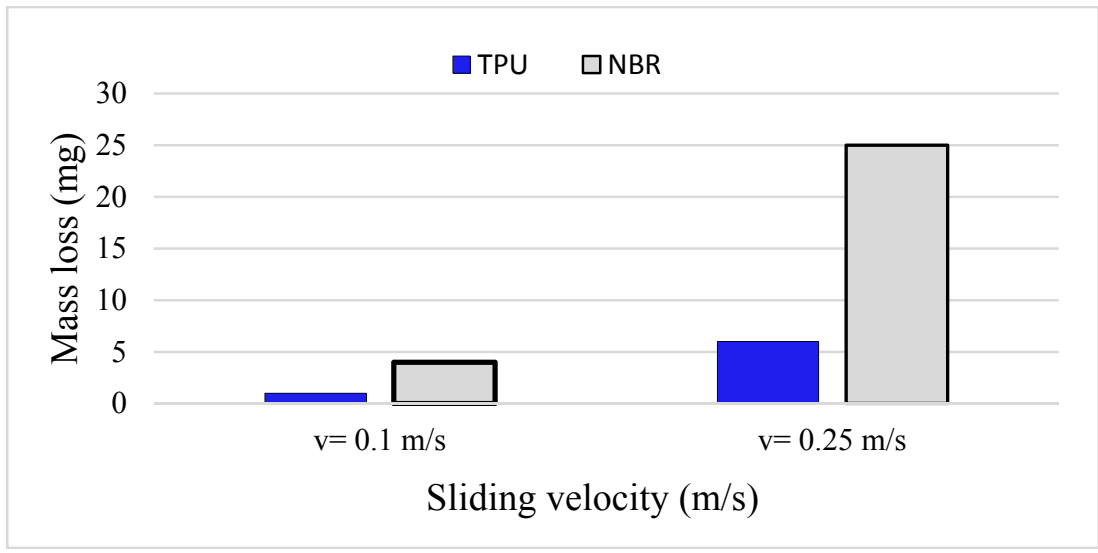

Figure 6: Mass loss of the elastomer samples at different velocity at sliding against a steel cylinder with $\mathrm{Ra}=0.5$.

\section{Conclusions}

In this work, the temperature rise of elastomeric surfaces sliding against steel parts under dry conditions was investigated. To this aim a high precision infrared thermos-camera was used to record the temperature field of the tribo-system during the tribological tests. Friction tests were carried out under a normal load of $15 \mathrm{~N}$, a stroke of $5 \mathrm{~mm}$, and various combinations of sliding velocities $(0.1$ and $0.25 \mathrm{~m} / \mathrm{s}$ ) and steel part roughness $(0.1$ and $0.5 \mu \mathrm{m})$. Furthermore, tests were carried out on two elastomers of different nature: TPU (Thermoplastic Polyurethane) and NBR (Nitrile Rubber). Even if their nature is different, both materials are very used in the sealing industry to manufacture seals for low velocities $(<0.5 \mathrm{~m} / \mathrm{s})$ and hydraulic applications.

Result showed that even if both materials present similar friction coefficients under the selected testing conditions the mass loss of the NBR is approximately 4 times greater than the mass loss of the TPU i.e. the wear resistance of the TPU is considerably better. Nevertheless, the TPU material presented also disadvantages such as instabilities in the friction curves due to vibrations during the tests. It is thought that the origin of these vibrations may be the great hardness of the material (93 Shore A). 
It was found that the effect of the sliding velocity plays a major role both in the generated friction and in the local heating that leads to a considerable temperature rise of the surfaces.

It can be concluded that friction and temperature rise increase monotonically with roughness of the steel counter-parts even if under the selected test conditions its even is almost negligible comparing to the effect of velocity.

\section{References}

[1] Conte, M., Pinedo, B. \& Igartua, A. Frictional heating calculation based on tailored experimental measurements. Tribology International, 74, 1-6, 2014.

[2] Conte, M., Pinedo, B. \& Igartua, A. Effect of contact temperature on the tribological behaviour of PTFE composites. New Developments in Polymer Composites Research, Nova publications, 2013.

[3] Conte M., Pinedo B. \& Igartua A. Frictional heating calculations for polymers. Surface effects and contact mechanics XI, Witpress, 2013.

[4] Kalin, M. Influence of flash temperatures on the tribological behaviour in low-speed sliding: a review. Materials Science and Engineering: A, 374(12), 390-397, 2004.

[5] Tzanakis, I., Conte, M., Hadfield, M. \& Stolarski, T. A. Experimental and analytical thermal study of PTFE composite sliding against high carbon steel as a function of the surface roughness, sliding velocity and applied load. Wear, 303(1-2), 154-168, 2013.

[6] Guha, D. \& Roy Chowdhuri, S. K. The effect of surface roughness on the temperature at the contact between sliding bodies. Wear, 197(1-2), 63-73, 1996.

[7] Conte, M. \& Igartua, A. Study of PTFE composites tribological behaviour, Wear, 296. 568-574, 2012. 